\title{
A Note on Orthography
}

Althougr Indonesian orthography has recently been modified slightly to correspond with the Malay, the present monograph retains the standard orthography for each language which was extant at the time of fieldwork (1969-70) and is utilized by the majority of written sources cited. However, several terms which have identical meaning and pronunciation in the Indonesian and Malay languages, but vary slightly in spelling, are given a single spelling, the Indonesian, throughout this text. 
This rationalization of conduct within the world, but for the sake of the world beyond, was the consequence of the concept or calling of ascetic Protestantism.

\section{Max Weber ${ }^{1}$}

If adequacy with respect to meaning is lacking, then no matter how high the degree of uniformity of process (whether overt or subjective) and how precisely its probability can be numerically determined, it remains an incomprehensible statistical probability. On the other hand, even the most perfect adequacy on the level of meaning has causal significance from a sociological point of view only insofar as there is some kind of proof for the existence of a probability that action in fact normally takes the course which has been held to be meaningful.

Max Weber ${ }^{2}$

1. Weber (1958, p. 154).

2. Weber (1947, p. 99; 1966, p. 11). This quotation differs in one particular from Parsons' 1947 translation, which reads: "No matter how high the degree of uniformity and how precisely its probability can be numerically determined, it is still an incomprehensible statistical probability, whether dealing with overt or subjective process." Weber's original is more literally translated as above: ". . uniformity of process (whether overt or subjective) . . ." which makes clearer the relation between "uniformity," "its," "overt or subjective," and "process." 